\title{
The Effects of Technical \\ Chlordane on Growth and Energy Metabolism of Streptococcus faecalis and Mycobacterium phlei: a Comparison with Bacillus subtilis
}

\author{
By R. WIDDUS, * P. W. TRUDGILL AND D. C. TURNELL \\ Department of Biochemistry and Agricultural Biochemistry \\ University College of Wales, Aberystwyth, $S Y 23{ }_{3} D D$
}

(Accepted for publication 10 August I97I)

S UM M A R Y

The growth of Mycobacterium phlei and Bacillus subtilis either on plate culture or in liquid medium is completely inhibited by technical chlordane at identical concentrations. NADH oxidation by membrane fragments and oxidative phosphorylation by crude extracts of $M$. phlei are only partially inhibited by incubation with chlordane at concentrations equal to or greater than the minimum levels required to completely inhibit growth.

Streptococcus faecalis is relatively insensitive to technical chlordane, bacteriostasis only being achieved at chlordane concentrations some five- to tenfold greater than those required with Bacillus subtilis and Mycobacterium phlei. The energy metabolism of $S$. faecalis is relatively simple since it contains neither a cytochromemediated electron transport chain nor a functional TCA cycle. Fermentation of Dglucose by soluble protein extracts, Na-K-ATPase and cation permeability of the plasma membrane are not sensitive to chlordane at concentrations that stop growth in liquid culture. Inhibition of fermentation by growing cultures, upon addition of chlordane, may be a reflection of the inhibition of biosynthetic reactions that recycle ATP.

\section{INTRODUCTION}

Organochlorine insecticides are inhibitory to the growth of Gram-positive bacteria (Gray \& Rogers, 1955; Duda, 1958; Lyr, 1969; Trudgill, Widdus \& Rees, I97 I). A range of sensitivity has been observed, Bacillus subtilis being prevented from growth by lesser chlordane concentrations than are required to produce similar results with Sarcina lutea and Streptococcus faecalis. Effects observed with chlordane treated B. subtilis include cessation of growth, inhibition and eventual cessation of respiration, loss of viability coupled with a delayed release of polymeric molecules from within the bacteria (Trudgill et al. 197I). Inhibition of whole-cell respiration is paralleled by inhibition of electron-transport capability of subcellular membrane fragments. Disruption of this and other membrane and mesosome associated phenomena has been postulated as being the primary cause of cessation of growth and loss of viability (Widdus, Trudgill \& Maliszewski, I97I).

In the present work extracts of Mycobacterium phle $i$ have been used to assess the possible importance of oxidative phosphorylation as a chlordane-sensitive metabolic activity. An attempt has also been made to assess the importance of the TCA cycle and electron transport as chlordane sensitive target sites by reference to Streptococcus faecalis. Though $S$. faecalis

* Present address: Centre for Theoretical Biology, State University at Buffalo, New York I4226, U.S.A. 
contains the respiratory components necessary for oxidative phosphorylation in the flavin region (Baum \& Dolin, 1965) it is devoid of cytochromes (Dolin, 1955) and is only capable of limited oxidative phosphorylation associated with NADH dehydrogenase (Gallin \& VanDemark, 1964; Faust \& VanDemark, 1970) when grown under aerobic conditions or provided with a suitable electron acceptor during anaerobic growth.

\section{METHODS}

Organisms. Streptococcus faecalis, strain I0 C I (NCIB866I) and Mycobacterium phlei (ATCC 354) were obtained from the respective culture collections. Stock cultures of $M$. phlei were maintained on nutrient agar. For $S$. faecalis the agar was fortified with $3 \%(\mathrm{w} / \mathrm{v})$ D-glucose and $5 \%(\mathrm{w} / \mathrm{v}) \mathrm{K}_{2} \mathrm{HPO}_{4}$. Cultures were incubated under static conditions at $37^{\circ}$ or, when fully aerobic conditions were required, in a New Brunswick Gyrotary shaker (New Brunswick Scientific Co., New Brunswick, New Jersey, U.S.A.) at $200 \mathrm{rev} . / \mathrm{min}$. and $37^{\circ}$. Growth in fermenter vessels was rendered anaerobic when required by passage of oxygenfree $\mathrm{N}_{2}$ through the medium.

Mycobacterium phlei was grown on nutrient agar or in liquid medium ( $45^{\circ}$ and $\left.\mathrm{I} 50 \mathrm{rev}.\right)$ min.) which contained (g./1.): proteose peptone, 13 ; sodium fumarate, $5 ; \mathrm{Na}_{2} \mathrm{HPO}_{4}$, I; $\mathrm{KH}_{2} \mathrm{PO}_{4}, 0.5 ; \mathrm{MgSO}_{4} .7 \mathrm{H}_{2} \mathrm{O}, 0.3 ; \mathrm{FeSO}_{4} .7 \mathrm{H}_{2} \mathrm{O}, 0.02$.

Harvesting and disruption of organisms. Bacteria were harvested and disrupted by the methods described by Widdus et al. (1971).

Measurement of bacterial growth. Growth of Streptococcus faecalis in liquid culture was followed as previously described (Trudgill et al. I971).

Addition of technical chlordane to aqueous growth media. Additions of chlordane to both liquid culture media and agar plates were made as described previously by Trudgill et al. (I97I).

Measurement of acid production by growing cultures of Streptococcus faecalis. Acid production was measured either by following the decrease in $\mathrm{pH}$ subsequent to the addition of a $10 \%(\mathrm{~V} / \mathrm{v})$ inoculum to $750 \mathrm{ml}$. of medium in a $\mathrm{I} 1$. fermenter pot on a Type $\mathrm{I}$ fermenter (L. H. Engineering, Stoke Poges, Buckinghamshire) or by addition of a $10 \%(\mathrm{v} / \mathrm{v})$ inoculum

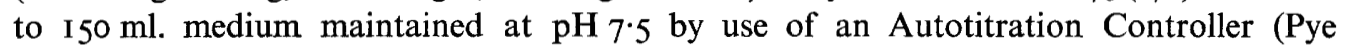
Unicam Ltd, Cambridge). In the latter case consumption of $1.5 \mathrm{~N}-\mathrm{KOH}$ was monitored manually at suitable time intervals. Growth media were maintained at $37^{\circ}$.

Preparation of crude extracts. Broken bacterial suspensions were centrifuged at $0^{\circ}$ and $20,000 \mathrm{~g}$ for $10 \mathrm{~min}$. The supernatant crude extract was subjected to further fractionation where required.

Preparation of high-speed supernatant fluid and membrane fraction. The supernatant fluid from the $20,000 \mathrm{~g}$ centrifugation was centrifuged at $125,000 \mathrm{~g}$ for $\mathrm{I} \mathrm{h}$. The high-speed supernatant fluid was decanted and the pellet, washed by resuspension in a suitable buffer followed by further centrifugation at $125,000 \mathrm{~g}$, was designated the crude membrane fraction.

Protein estimations. The protein content of extracts was determined by the modified biuret procedure of Gornall, Bardawill \& David (I949).

Measurement of acid production by Streptococcus faecalis extracts. The fermentation of D-glucose by extracts of $S$. faecalis was monitored by the controlled addition of I mM$\mathrm{NaOH}$ to an unbuffered reaction system containing the necessary additional reaction components. The $\mathrm{pH}$ was maintained at 7.5 by the Autotitration Controller and alkali consumption monitored manually at suitable time intervals.

NADH oxidase activities. NADH oxidases and peroxidases of high-speed supernatant 
fluid and membrane fractions of Streptococcus faecalis were assayed by following the fall in $E_{340}$ when $0.5 \mu$ mole of NADH was added to a suitable amount of protein contained in a final vol. of $3 \mathrm{ml}$. $0 \cdot 0 \mathrm{I}$ M-tris- $\mathrm{HCl}$ buffer, $\mathrm{pH} 8 \cdot 0$. Cytochrome chain mediated NADH oxidation by crude extracts or membrane fractions from Mycobacterium phlei was measured by following the stimulation of $\mathrm{O}_{2}$ consumption that occurred when $2 \mu$ mole NADH were added to a $3 \mathrm{ml}$. reaction volume that contained I to $5 \mathrm{mg}$. of protein and appropriate buffer. Determinations were carried out in a conventional Warburg apparatus or with a Clark-type oxygen electrode in an agitated and thermostatically controlled vessel (Oxygen Monitor, model 53; Yellow Springs Instrument Co. Inc., Yellow Springs, Ohio, U.S.A.).

Measurement of oxidative phosphorylation. Oxygen consumption by extracts of Mycobacterium phlei was measured as described and residual inorganic phosphate estimated by the method of Fiske \& SubbaRow (1925) subsequent to the denaturation of protein by addition of $10 \%(\mathrm{w} / \mathrm{v})$ trichloroacetic acid and its removal by centrifugation.

Adenosine triphosphatase (ATPase) activity. Na-Ka-ATPase of Streptococcus faecalis was assayed according to the method of Harold, Baarda, Baron \& Abrams (1969).

\section{RESULTS}

\section{Mycobacterium phlei}

Effects of chlordane on growth of Mycobacterium phlei. The growth of $M$. phlei on nutrient agar plates was inhibited by technical chlordane added as a surface film and displayed the same sensitivity as Bacillus subtilis (Table 1). Because of the granular growth characteristics of $M$. phlei in liquid culture it was not possible effectively to monitor growth by following turbidity at $580 \mathrm{~m} \mu$. Accordingly the effects of chlordane on the growth of $M$.

Table I. The sensitivity of Mycobacterium phlei growing on agar plates to technical chlordane: a comparison with Bacillus subtilis, Streptococcus faecalis and Sarcina lutea

\begin{tabular}{lcccc} 
& \multicolumn{4}{c}{ Chlordane $\left(\mathrm{mg} . / \mathrm{cm}^{2}{ }^{2}\right)$} \\
\cline { 2 - 5 } Micro-organism & $\mathrm{I} \cdot 0$ & $0 \cdot \mathrm{I}$ & $0 \cdot 0 \mathrm{I}$ & Vapour* \\
M. phlei & - & - & - & - \\
B. subtilis $\dagger$ & - & - & - & - \\
S. faecalis $\dagger$ & - & - & + & - \\
S. lutea $\dagger$ & - & - & $(+)$ & -
\end{tabular}

+ , Growth not inhibited; $(+)$, growth slightly inhibited; - , growth severely or completely inhibited.

* A drop of chlordane was placed in the lid of each inverted Petri dish.

$\uparrow$ Data from Trudgill et al. (1971).

Table 2. Inhibition of growth of Mycobacterium phlei in liquid culture by technical chlordane

Erlenmeyer flasks containing $50 \mathrm{ml}$. medium were inoculated with $M$. phlei followed by additions as indicated. After $72 \mathrm{~h}$. growth at $45^{\circ}$ on a gyrotary shaker, cultures were harvested on tared $5 \mu$ pore size membrane filters and dried to constant weight.

\section{Addition}

Acetone (control)

Chlordane (5 p.p.m.)

Chlordane (Io p.p.m.)

Chlordane (20 p.p.m.)

Chlordane (Ioo p.p.m.)

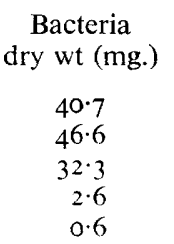


phlei were assayed by harvesting cells from experimental and control culture flasks on predried and weighed membrane filters. The dry weights of bacteria obtained (Table 2) confirm the growth sensitivity of $M$. phlei to technical chlordane as being of the same order as that of Bacillus subtilis. It was therefore considered that $M$. phlei was an ideal organism in which to test the effects of chlordane on oxidative phosphorylation since sub-cellular systems of $M$. phlei capable of catalysing effective oxidative phosphorylation with high $\mathrm{P} / \mathrm{O}$ ratios have been well described by Brodie \& Gray (1956). The NADH oxidase activity of crude membrane preparations is chlordane sensitive as shown in Table 3. The inhibition obtained was

\section{Table 3. Effects of technical chlordane on $N A D H$ oxidase activity of crude membrane preparations from Mycobacterium phlei}

NADH oxidase activity was measured as described in the Methods. The oxygen monitor vessel contained $2 \mu$ mole NADH, 2 mg. crude membrane protein and $280 \mu$ mole tris- $\mathrm{HCl}$ buffer $\mathrm{pH} 7 \cdot 4$. Reactions were followed at $37^{\circ}$.

Addition

None

Acetone

Chlordane (20 p.p.m.)

Chlordane (Ioo p.p.m.)
NADH oxidase* activity ( $\mu$ mole $\mathrm{O}_{2} / \mathrm{mg}$. protein $/ \mathrm{h}$.)

$$
\begin{aligned}
& 2 \cdot 4 \\
& I \cdot 75 \\
& I \cdot I 4 \\
& 0 \cdot 74
\end{aligned}
$$

Inhibition
$(\%)$
-
0
35
58

* Assay systems were incubated with acetone or chlordane for $1 \mathrm{~h}$. at $37^{\circ}$ prior to addition of NADH.

Respiration was measured with the oxygen monitor as described in the Methods section. Reaction systems contained the following: $\mathrm{KH}_{2} \mathrm{PO}_{4}, 5 \mu \mathrm{mole} ; \mathrm{MgCl}_{2}$, Io $\mu$ mole; D-mannose, $20 \mu$ mole; ADP, $5 \mu$ mole; KF, $20 \mu$ mole; hexokinase, $2 \cdot 8$ units and Io to $15 \mathrm{mg}$. crude extract protein. Volumes were made to $3 \mathrm{ml}$. with tris/ $\mathrm{HCl}$ buffer $\mathrm{pH} 7.4$ and reactions started by addition of $2 \mu$ mole NADH.

Reaction system

(a) NADH absent Complete Complete

(b) Complete Complete Complete
Addition

$10^{-3} \mathrm{M}$-dinitrophenol $\uparrow$

Acetone

Chlordane (20 p.p.m.)

Chlordane (I00 p.p.m.)

$\begin{array}{cl}\begin{array}{c}P_{i} \\ \text { esterified } \\ (\mu \text { mole })\end{array} & \begin{array}{l}\text { P/O } \\ \text { ratio }\end{array} \\ 0.00 & 0 \\ \mathrm{I} .62 & \mathrm{I} .87 \\ 0.00 & 0 \\ \mathrm{I} .05 & \mathrm{I} .05 \\ 0.75 & 0.70 \\ 0.57 & 0.54\end{array}$

* Reactions were stopped after consumption of the amounts of $\mathrm{O}_{2}$ indicated. Dinitrophenol caused no significant change in the rate of $\mathrm{O}_{2}$ consumption. Addition of chlordane at 20 and I00 p.p.m. caused 22 and $45 \%$ inhibition of the rate of $\mathrm{O}_{2}$ uptake, respectively, in comparison with the acetone control. $(a)$ and $(b)$ were conducted with different bacterial extracts.

in general less than that observed with membrane preparations from $B$. subtilis (Trudgill et al. I971) and showed some variation with the age of the membrane preparation. Oxidative phosphorylation by crude extract with $\mathrm{NADH}$ as electron donor yielded $\mathrm{P} / \mathrm{O}$ ratios in the range of 0.8 to $\mathrm{I} \cdot 8$ and was completely inhibited by $\mathrm{IO}^{-3} \mathrm{M}$-dinitrophenol. Incubation of crude extract with chlordane at 20 and 100 p.p.m. resulted in significant inhibition of oxidative phosphorylation (Table 4), though the data show that under the experimental conditions employed, electron transport by the cytochrome chain is more chlordane sensitive than the phosphorylation step so that oxidative phosphorylation cannot be regarded as a particularly sensitive target site. 


\section{Streptococcus faecalis}

Sensitivity of growth and fermentation by Streptococcus faecalis to technical chlordane. The growth of $S$. faecalis on nutrient agar plates fortified with D-glucose has been shown to be sensitive to the presence of technical chlordane though the organism is not so sensitive as other Gram-positive bacteria, for example Bacillus subtilis (ATCC9372) (Trudgill et al. 1971). This lower sensitivity of $S$. faecalis was demonstrated during growth in liquid culture (Fig. I), 200 p.p.m. chlordane being required to cause a near complete inhibition of cell growth $\left(+\triangle E_{580}\right.$ ) while complete inhibition of growth of $B$. subtilis cultures at a similar cell density was achieved at 20 p.p.m. chlordane. The loss of viability and lysis observed with B. subtilis (Trudgill et al. 197I) were not evident when $S$. faecalis was treated with 200 p.p.m. chlordane. Concomitant with the near cessation of growth of $S$. faecalis which was usually delayed until some $30 \mathrm{~min}$. after the addition of chlordane, a marked inhibition of Dglucose fermentation was suggested by the abrupt inflexion in the $\mathrm{pH}$ curve.

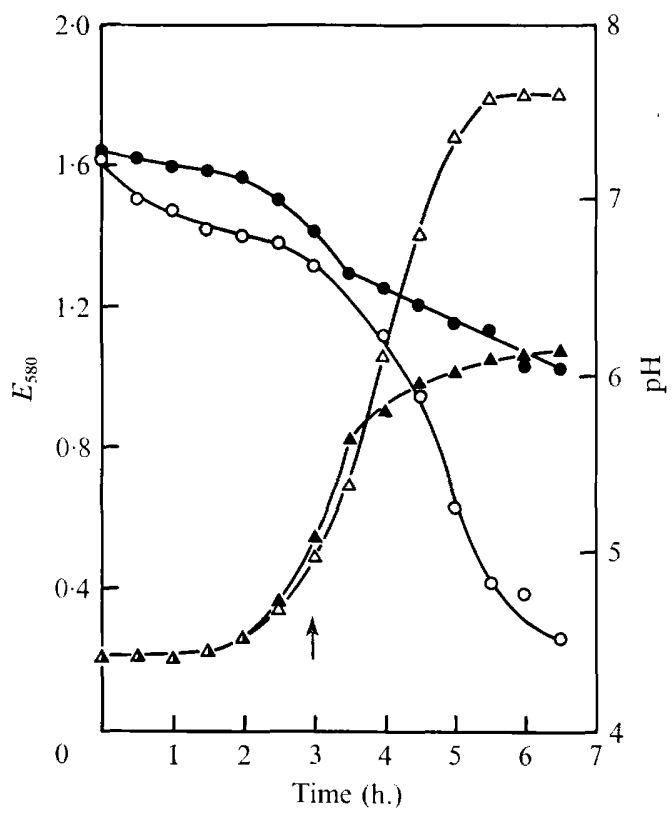

Fig. I

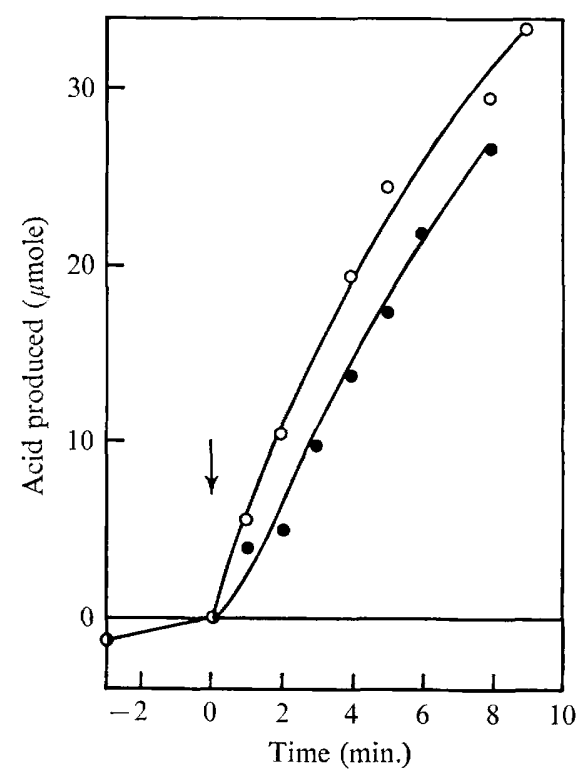

Fig. 2

Fig. I. Effect of technical chlordane on growth of Streptococcus faecalis and medium pH profile under anaerobic conditions. $\triangle, \Delta, E_{580} ; 0, \boldsymbol{0}, \mathrm{pH}$ of growth medium. Chlordane (200 p.p.m.) was added to test experiment $(\Delta, \boldsymbol{\theta})$ and acetone to the control $(\triangle, O)$ at the time indicated by the arrow.

Fig. 2. The effect of technical chlordane on glycolysis by high-speed supernatant fluid from Streptococcus faecalis. Reaction mixtures on the autotitration controller in deionized water, final vol. $10 \mathrm{ml}$., pH 7.5 contained: ADP, $40 \mu$ mole; $\mathrm{KH}_{2} \mathrm{PO}_{4}, 40 \mu$ mole; $\mathrm{NAD}, 5 \mu$ mole; ATP, $5 \mu$ mole; $68 \mathrm{mg}$. protein. Reaction was started by the addition of glucose $(20 \mu \mathrm{mole})$ and acid production followed as described in Methods. High-speed supernatant fluid was incubated for $\mathrm{I} h$. with acetone as control (O) and chlordane at 200 p.p.m. (๑).

The decreased fermentation rate that occurred with growing cultures was not reproduced with a cell-free glycolytic system maintained at $\mathrm{pH} 7 \cdot 5$. When acid production from D)-glucose was monitored in the presence of the necessary cofactors, incubation of crude 
extract with 200 p.p.m. technical chlordane for I h. did not inhibit glycolysis (Fig. 2). This suggested that inhibition of glycolysis observed with growing cultures was not due to any direct effect of the chlordane upon the glycolytic enzymes but rather that it might be due to inhibition of associated or coupled phenomena in whole cell metabolism. Such phenomena include biosynthetic reactions that utilize ATP and the cation transporting ATPase present in the plasma membrane (Harold et al. 1969). It was also possible that such effects might be compounded by modification of membrane permeability and uncoupling of cation transport from membrane ATPase so that the intracellular $\mathrm{pH}$ was not maintained more alkaline than

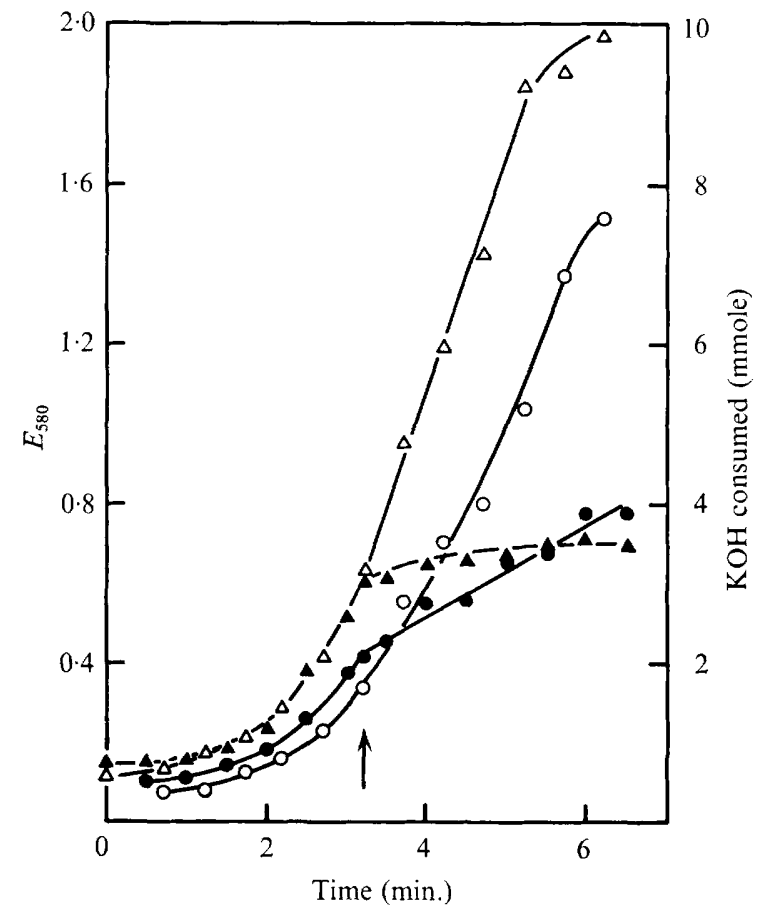

Fig. 3. Effect of technical chlordane on growth and acid production by Streptococcus faecalis maintained at constant $\mathrm{pH}$. Acid production by $S$. faecalis growing at $37^{\circ}$ was followed by monitoring addition of $\mathrm{I} \cdot 5 \mathrm{~N}-\mathrm{KOH}$ required to maintain the culture at $\mathrm{pH} 7.5$ as described in Methods. $\triangle, \Delta, E_{580} ; \bigcirc, \bigcirc$ addition of alkali. Chlordane (200 p.p.m.) was added to the test culture $(\boldsymbol{\Lambda}, \boldsymbol{\bullet})$ and acetone to the control $(\triangle, O)$ at the time indicated by the arrow.

that of the growth medium by energy-dependent proton excretion (Harold, Pavlasova \& Baarda, 1970). Under the experimental conditions (Fig. I) a decrease of intracellular $\mathrm{pH}$ value to that of the growth medium at the time of chlordane addition would itself contribute to the inhibition of glycolysis. Since a similar pattern of inhibition of glycolysis was observed when chlordane at 200 p.p.m. was administered to a growing culture maintained at $\mathrm{pH} 7.5$ on the $\mathrm{pH}$ stat (Fig. 3) lack of control of intracellular $\mathrm{pH}$ as a result of changes in membrane permeability was not a significant factor in the inhibition of glycolysis.

Effect of chlordane on Na-K-ATPase of Streptococcus faecalis. The membrane-bound ATPase of $S$. faecalis is involved in the energy dependent transport of cations across the plasma membrane (Abrams, 1960; Zarlengo \& Schultz, I966; Harod et al. 1969). Since this enzyme hydrolyses ATP generated at substrate level during glycolysis, inhibition of ATPase may interrupt glycolysis by restricting the recycling of ATP as was observed with $N, N$ - 
dicyclohexylcarbodiimide (Harold et al. 1970). Incubation of crude membrane preparations from $S$. faecalis with 200 p.p.m. technical chlordane for $\mathbf{I}$ h. did not inhibit Na-K-ATPase, suggesting that the observed inhibition of glycolysis was not due to interference with this ATP recycling reaction.

Effect of chlordane on NADH oxidizing enzymes of Streptococcus faecalis. The mixture of NADH oxidases and peroxidases responsible for the respiration of intact $S$. faecalis, devoid of haeme pigments, under aerobic conditions have been variously reported as being coupled to oxidative phosphorylation (Gallin \& VanDemark, 1964) and not involved in generation of ATP (Bryn-Jones \& Whittenbury, 1969). In view of the possibility that these enzymes may be of importance in the energy metabolism of aerobically grown $S$. faecalis the effect of technical chlordane was investigated as described in the Methods section. Incubation of subcellular fractions for $\mathrm{I} h$. with 200 p.p.m. chlordane inhibited NADH oxidation by a somewhat variable amount which ranged from 6 to $29 \%$ from preparation to preparation. Membrane bound activity was not significantly more sensitive than the soluble enzymes.

\section{DISCUSSION}

Attempts to elucidate the nature of the cyclodiene insecticide sensitivity of Bacillus subtilis (ATCC 9372) that is responsible for inhibition of growth and respiration and for loss of viability have been only partially successful (Trudgill et al. 197I; Widdus et al. 197I). Inhibition of bacterial respiration is paralleled by inhibition of NADH oxidase of isolated membrane fragments, indicating that, in common with other biochemical systems studied (Morrison \& Brown, 1954; Colvin \& Phillips, 1968; Wineley \& San Clemente, 1970), organized lipid-containing membranous structures are targets that attract hydrophobic cyclodiene insecticides. However, because cessation of bacterial growth precedes the complete inhibition of respiration it may be argued that the observed effects upon respiration are secondary phenomena (Widdus et al. 197I). The possibility also exists that the phosphorylation steps coupled to cytochrome mediated electron transport may be chlordane sensitive. To test this suggestion crude extract preparations from Mycobacterium phlei (ATCC 354) that are capable of effective oxidative phosphorylation (Brodie \& Gray, 1956) were used. Though growing cultures of $M$. phlei exhibited the same sensitivity to technical chlordane as $B$. subtilis (Tables I, 2) oxidative phosphorylation by in vitro systems is only partially inhibited and displays a sensitivity no greater than that shown by NADH oxidase of uncoupled membrane fragments (Tables 3,4 ). In $M$. phlei, and perhaps $B$. subtilis, neither electron transport nor coupled oxidative phosphorylation would appear to be a critically disrupted primary site of chlordane action.

By comparison with Bacillus subtilis the relative insensitivity of Streptococcus faecalis could be attributed to a lack of cytochromes and TCA cycle in the latter organism. Such an argument is reinforced by the studies of Nelson \& Williams (197I) who showed that aerobically grown Saccharomyces cerevisiae displayed a time-dependent inhibition of respiration in the presence of chlordane. Only when the organism was grown aerobically on nonfermentable substrates, with an obligatory requirement for oxidative phosphorylation, did chlordane inhibit growth. The view that electron transport, oxidative phosphorylation and the TCA cycle are primary targets of cyclodiene action is however probably an oversimplification of the situation with respect to bacteria and does not explain the variation in sensitivity exhibited by organisms within the same genus and even different strains of the same species (Fletcher \& Bollen, I954; Trudgill et al. 1971).

Sarcina lutea, which has a cytochrome complement very similar to that of Bacillus subtilis 
(Gel'man, Lukoyanova \& Ostrovskii, 1967), was the least sensitive of the Gram-positive organism tested (Trudgill et al. 1971). Clearly other factors such as differences in cell wall structure and composition (the factor responsible for resistance of Gram-negative bacteria) and, in this case perhaps the association of carotenoids with the plasma membrane (Mathews \& Sistrom, 1959), might play a role in conferring a degree of resistance to the effects of technical chlordane.

Technical chlordane is bacteriostatic to Streptococcus faecalis but only at relatively high concentrations (Fig. I). The effect of chlordane is not due to inhibition of the membranebound ATPase with a resulting disruption of energy-dependent ion transport across the plasma membrane nor does it appear to involve any aspect of the energy metabolism of glucose-grown bacteria (Fig. 2).

The inhibition of glycolysis by whole bacteria that accompanies virtual cessation of growth (Fig. 3) may reflect the inhibition of energy-dependent biosynthetic processes with a consequent decrease in the rate of turnover of ATP imposing a limitation on glycolysis. Our failure to show inhibition of glycolysis in high-speed supernatant fluids is compatible with the relative insensitivity of soluble enzymes as reported for Bacillus subtilis (Widdus et al. 1971).

As previously suggested (Widdus et al. 197I) and not contradicated by the data presented here, the cyclodiene insecticides probably exert their effects by deformation of membrane integrity the primary results of which are a cessation of membrane associated aspects of cell division and biosynthesis while effects upon energy metabolism may appear as closely associated secondary phenomena.

Our thanks are due to the Velsicol Chemical Corp. for generously supplying us with technical chlordane, to the Science Research Council for support for one of us (R.W.) with a studentship during the course of this work, to Mr J. S. Rees for expert technical assistance and to Mrs G. Roberts for typing the manuscript.

\section{REFERENCES}

ABrams, A. (I960). Metabolically dependent penetration of oligosaccharides into bacterial cells and protoplasts. Journal of Biological Chemistry 235, $128 \mathrm{I}-1285$.

BaUm, R. H. \& Dolin, M. I. (1965). Isolation of 2-solanesyl-I,4-napththoquinone from Streptococcus faecalis Io C I. Journal of Biological Chemistry 240, 3425-3433.

Brodie, A. F. \& GraY, C. T. (1956). Phosphorylation coupled to oxidation in bacterial extracts. Journal of Biological Chemistry 219, 853-862.

BRYN-JONES, D. G. \& WhitTENBURY, R. (1969). Haematin-dependent oxidative phosphorylation in Streptococcus faecalis. Journal of General Microbiology 58, 247-260.

Colvin, H. J. \& Phillips, A. T. (1968). Inhibition of electron transport and cholinesterases by endrin. Bulletin of Environmental Contamination and Toxicology 3, 106-I 15.

Dolin, M. I. (1955). The DPNH-oxidizing enzymes of Streptococcus faecalis. Archives of Biochemistry \& Biophysics 55, 415-435.

DudA, A. (1958). The effects of hexachlorocyclohexane and chlordane on soil microflora. Acta microbiologica polonica $7,237-244$.

Faust, P. J. \& VANDEMARK, P. J. (1970). Phosphorylation coupled to NADH oxidation with fumarate in Streptococcus faecalis Io C I. Archives of Biochemistry and Biophysics 137, 392-398.

FisKe, C. H. \& SubBaRow, Y. (I925). The colorimetric determination of phosphorus. Journal of Biological Chemistry 66, 375.

FLeTCHER, D. W. \& Bollen, W. B. (1954). The effects of aldrin on soil micro-organisms and some of their activities related to soil fertility. Applied Microbiology 2, 349-354.

Gallin, J. I. \& Vandemark, P. J. (I964). Evidence for oxidative phosphorylation in Streptococcus faecalis Io C I. Biochemical and Biophysical Research Communications 17, 630-635. 
Gel'man, N. S., Lukoyanova, M. A. \& Ostrovskit, D. N. (1967). Respiration and Phosphorylation in Bacteria, p. 9I. Edited by G. B. Pinchot. New York: Plenum Press.

Gornall, A. G., Bardawill, C. J. \& David, M. M. (1949). Determination of serum proteins by means of the biuret reaction. Journal of Biological Chemistry 177, 75I-766.

GrAY, P. H. H. \& Rogers, C. G. (1955). Effects of benzene hexachloride on soil micro-organisms. Canadian Journal of Microbiology I, 312-318.

Harold, F. M., BaARda, J. R., Baron, C. \& Abrams, A. ( I969). Inhibition of membrane bound ATPase and cation transport of Streptococcus faecalis by $N, N$-dicyclohexylcarbodiimide. Journal of Biological Chemistry 244, 226I-2268.

Harold, F. M., Pavlasova, E. \& BaArda, J. R. (1970). A transmembrane pH gradient in Streptococcus faecalis: origin, and dissipation by proton conductors and $N, N$-dicyclohexylcarbodiimide. Biochemica et biophysica acta 196, 235-244.

LYR, H. (1969). On the mechanism of action of $\delta$-hexachlorocyclohexane. Zeitschrift für Allgemeine Mikrobiologie 9, 197-204.

Mathews, M. M. \& Sistrom, W. R. (1959). Intracellular location of carotenoid pigments and some respiratory enzymes in Sarcina lutea. Journal of Bacteriology 78, 778-787.

Morrison, P. E. \& BRown, A. W. A. (I954). The effects of insecticides on cytochrome oxidase obtained from the American cockroach. Journal of Economic Entomology 47, 723-730.

Nelson, B. D. \& Williams, C. (1971). Action of cyclodiene pesticides on oxidative metabolism in the yeast Saccharomyces cerevisiae. Journal of Food and Agricultural Chemistry 19, 339-34I.

Trudgill, P. W., Widdus, R. \& Rees, J. S. (197I). Effects of organochlorine insecticides on bacterial growth, respiration and viability. Journal of General Microbiology 69, I-I 3.

Widdus, R., Trudgill, P. W. \& Maliszewski, M. J. (I97I). The effects of technical chlordane on energy metabolism of Bacillus subtilis. Journal of General Microbiology 69, I 5-22.

Wineley, C. L. \& SAN Clemente, C. L. (1970). Effects of pesticides on nitrite oxidation by Nitrobacter agilis. Applied Microbiology 19, 214-219.

Zarlengo, M. H. \& SChultz, S. G. (1966). Cation transport and metabolism in Streptococcus faecalis. Biochimica et biophysica acta 126, 308-320. 